\title{
Thoracoscopy: assessment of a physician service and comparison of a flexible bronchoscope used as a thoracoscope with a rigid thoracoscope
}

\author{
A C DAVIDSON, R J GEORGE, C D SHELDON, G SINHA, B CORRIN, D M GEDDES \\ From the London Chest Hospital
}

ABSTRACT The practicality of physicians performing thoracoscopy for diagnostic purposes was assessed in 30 patients with pleural effusions of unknown cause. A rigid thoracoscope was compared with a fibreoptic bronchoscope used as a flexible thoracoscope and the diagnostic adequacy of biopsy specimens obtained with the two instruments assessed. The two instruments were inserted by a physician in the bronchoscopy suite using local anaesthesia. The procedure proved safe, acceptable, and diagnostically effective. The rigid thoracoscope proved a more satisfactory instrument but the fibreoptic bronchoscope, with minor adaptations, may be used for thoracoscopy.

\section{Introduction}

Introduced originally for diagnostic purposes, ${ }^{1}$ thoracoscopy gained widespread use for lysis of tuberculous pleural adhesions, falling into disuse with the introduction of effective chemotherapy. More recently, however, its diagnostic usefulness, particularly in cases of pleural effusion of unknown cause, has led to its reintroduction..$^{2-5}$ Various instruments and anaesthetic techniques have been used. ${ }^{2}$ Most often a specially designed rigid thoracoscope is employed, thoracic surgeons usually favouring general anaesthesia and physicians local anaesthesia. ${ }^{6-8}$ The fibreoptic bronchoscope has also been used as a thoracoscope, some claiming it to be technically superior to rigid instruments ${ }^{1011}$ but others finding it unsatisfactory. ${ }^{1213}$ Only one group has compared the two instruments? few patients were studied and difficulty was experienced in histological interpretation of the smaller specimens obtained with the fibreoptic bronchoscope.

The aims of the present study were, firstly, to assess the practicality of thoracoscopy performed by physicians in the bronchoscopy suite using local anaesthesia and, secondly, to compare the diagnostic effectiveness of the fibreoptic bronchoscope used as a flexible thoracoscope with that of the rigid thoracoscope.

Address for correspondence: Dr A C Davidson, Medical Unit, St Thomas's Hospital, London SE1. (Reprints will not be available.)

Accepted 15 December 1987

\section{Methods}

From May 1985 to July 1986 thoracoscopy was performed by a physician on 30 consecutive patients presenting to the London Chest Hospital with pleural effusion of unknown cause. The first 10 examinations were carried out with a rigid thoracoscope (a forward looking Storz thoracoscope of $9 \mathrm{~mm}$ external diamater) alone. Subsequently both the rigid thoracoscope and a fibreoptic bronchoscope (Pentax FB 19H, external diameter $5.9 \mathrm{~mm}$ ) were used.

The indications for thoracoscopy were conventional. Patients underwent the procedure when the cause of the pleural effusion remained unclear after aspiration (the fluid being sent for cytological, microbiological, and immunological examination) and a successful Abrams punch pleural biopsy. Other appropriate investigations-for example, bronchoscopy or lymph node biopsy-had also to have yielded negative results before thoracoscopy was undertaken. A further requirement was that the patient had to be considered fit for thoracoscopy. To assess this, spirometry was performed and, if hypoxaemia was suspected, arterial blood gases were sampled. The patient had to be able to lie comfortably without respiratory distress. No patient was considered unfit for the procedure during the course of the study.

Patients were admitted for 24 hours unless already under inpatient investigation. The procedure was explained and consent obtained. Before thoracoscopy $100-200 \mathrm{ml}$ pleural fluid was aspirated and replaced 
with a similar volume of air. A decubitus radiograph with the affected side uppermost was taken to determine the presence of adhesions or loculations and to assess the degree of pleural thickening. Premedication consisted of intramuscular atropine $(600 \mu \mathrm{g})$ and papaveretum (10-20 mg). Thoracoscopy was performed in the bronchoscopy suite, usually at the end of a routine bronchoscopy list, with the patient lying in the lateral decubitus position. An electrocardiograph was attached for monitoring purposes and intravenous diazepam $(5-20 \mathrm{mg})$ was given as necessary. Oxygen was supplied by mask or nasal prongs if arterial oxygen tension had been found to be less than $9.3 \mathrm{kPa}$ or if respiratory distress occurred during the procedure. On some occasions an ear oximeter (Biox III) was also used for monitoring purposes.

The rigid thoracoscope and the fibreoptic instrument were sterilised by cold immersion in $2 \%$ glutaraldehyde for at least 30 minutes. ${ }^{14}$ The operator and assistant scrubbed and wore surgical mask, gown, and gloves. After skin preparation $20 \mathrm{ml} 1 \%$ lignocaine were infiltrated between the 6 th and the 7 th or the 7 th and the 8th ribs in the posterior axillary line down to and including the parietal pleura, aspiration of air confirming pleural puncture. A stab incision was then made and a track created by blunt dissection with forceps. The Storz trocar was inserted and the pleural cavity opened to atmospheric pressure, any remaining pleural fluid being aspirated. A full examination of the pleural cavity was then made and biopsy specimens of parietal and visceral pleura were taken as appropriate under direct vision. After the first 10 examinations the pleural space was examined with both the rigid thoracoscope and the flexible bronchoscope in random order. The bronchoscope was introduced through the Storz trocar (after removal of the flap valve assembly) in the first 10 cases and through a size 24 Argyle chest drain acting as a sheath for the bronchoscope $^{89}$ in three cases. In the remaining patients the bronchoscope was passed through a Maligar trocar.

All 30 patients were examined by one operator (ACD), who had assisted at several "surgical" thoracoscopies before undertaking "medical" thoracoscopy. In the case of the 20 patients examined with both instruments, care was taken to select equally abnormal areas for biopsy. Multiple (5-7) biopsy samples were taken with each instrument to avoid any bias toward one or other technique in the assessment of the diagnostic usefulness of the two instruments.

At the end of the procedure our initial practice was to introduce an Argyle drain through the thoracoscopy incision and confirm lung expansion radiographically before removal of the tube. Since routine drainage may increase the risk of infection in the pleural space, ${ }^{515}$ we subsequently removed the Argyle drain as soon as air was no longer being expelled by coughing-that is, within a few minutes. When inspection had suggested malignancy or when the effusion $\frac{C}{0}$ had previously reaccumulated rapidly, drainage was $\frac{\bar{\omega}}{\bar{c}}$ continued to allow a tetracycline pleuradesis to be $\vec{\sigma}$ performed (with $500 \mathrm{mg}$ tetracycline dissolved in $50 \mathrm{ml} \cong$ saline with a dwell time of two hours and routine कै suction for 24 hours). Drainage was also maintained if $\vec{\circ}$ adhesions or visceral pleural thickening were likely to $\overrightarrow{\vec{A}}$ limit full expansion, $10-20 \mathrm{~cm} \mathrm{H}_{2} \mathrm{O}$ negative pleural $\vec{\sigma}$ pressure being applied. In all cases a radiograph was taken within 24 hours. The patients were reviewed at $\times$ monthly intervals or, in cases of secondary referral, the $\hat{\omega}$ referring physician was contacted for information on follow up. A final diagnosis was made in the light of the biopsy findings and the subsequent clinical course. $O$

The specimens obtained with the two instruments were processed separately. They were fixed in $10 \% \vec{\rightarrow}$ neutral buffered formalin, embedded first in agar to prevent tissue loss and then processed automatically to paraffin. Sections 3-5 $\mu$ m thick were cut at three levels, $\stackrel{\odot}{\varnothing}$ care being taken to ensure that all the embedded tissue 9 was sampled. Haematoxylin and eosin staining was performed routinely and immunocytochemical staining, with the use of monoclonal antisera to carcinoembryonic antigen, cytokeratin, and human milk fat globulins 1 and 2, was performed if indicated. Reports $\stackrel{\unrhd}{\perp}$ on the fibreoptic specimens were made independently $\overrightarrow{\vec{A}}$ of those on the rigid specimens but a combined report $\frac{3}{3}$ was issued for clinical use. Subsequently all sections were examined in random order by a second pathologist without knowledge of biopsy method or clinical data.

\section{Results}

Adequate pleural tissue was obtained in all but one $\delta$ case, in which access was limited by multiple adhesions and only normal lung tissue was obtained. The 을 histological diagnosis in the remaining 29 patients was $\rightarrow$ adenocarcinoma in five, mesothelioma or probable mesothelioma in 13, suspicion of malignancy in two, $\widetilde{\sim}$ tuberculosis in one, and chronic non-specific pleurisy in eight. The final diagnosis subsequently proved to be $N$ malignancy in all of the cases considered to be $\mathrm{\omega}$ malignant after thoracoscopy, although a necropsy $\mathcal{O}$ was not performed in all cases. Of the eight cases of 0 non-specific pleurisy, the final diagnosis proved to be $\mathbb{\Phi}$ malignancy in three (adenocarcinoma 2 , meso- ? thelioma 1) and probable mesothelioma in a fourth. In only four cases was the outcome benign, Mycobacterium tuberculosis subsequently being cultured from $\stackrel{\odot}{\stackrel{\oplus}{\oplus}}$ the biopsy material in one patient. Thoracoscopy $\mathbb{Q}$ therefore provided diagnostic material or specimens $\overline{-}$ correctly negative for malignancy in 23 patients, suggested malignancy in two, was unhelpful in one (no 
Thoracoscopy: assessment of physician service and comparison of flexible bronchoscope with rigid thoracoscope 329

Histology reports on biopsy specimens obtained with the rigid and fibreoptic instruments and comparison with the clinical outcome in the 20 patients examined with both instruments

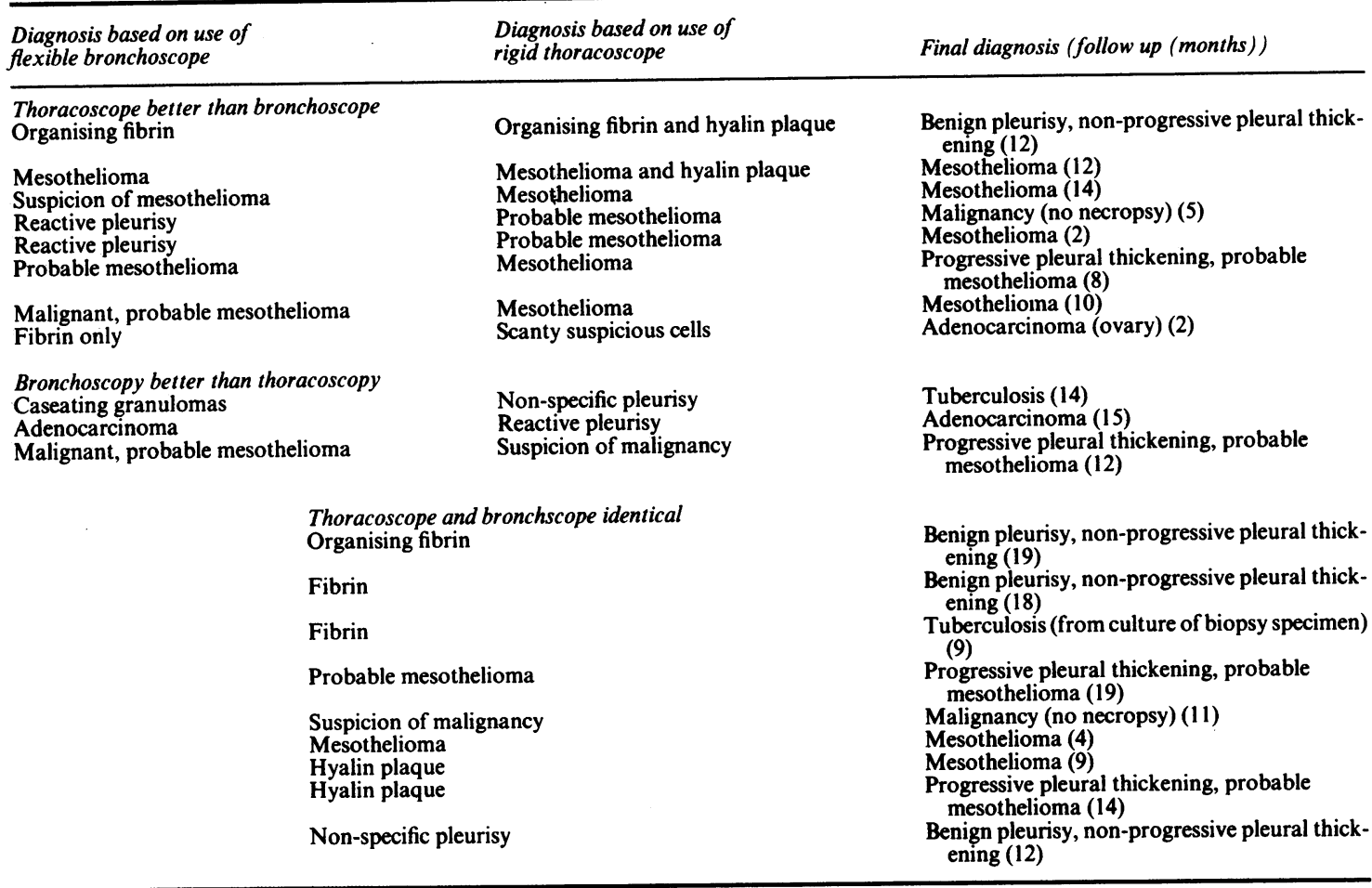

Specimens examined in random order and in ignorance of clinical history and thoracoscopic appearances.

biopsy specimen), and gave a false negative result in four.

The macroscopic appearances at thoracoscopy proved unreliable. In three cases of mesothelioma the thoracoscopic appearances had suggested asbestos pleural plaques with an apparently benign pleurisy, and in another three cases the histological appearance of the pleura and subsequent clinical course favoured a benign condition when visual inspection had suggested malignancy.

When biopsy specimens obtained with the two instruments were compared, the histological features were considered identical in nine cases, the specimens obtained with the fibreoptic bronchoscope more informative in three, and those obtained from the rigid thoracoscope more informative in eight (table). The specimens obtained with the rigid instrument were larger and sometimes provided additional information - for example, both mesothelioma and benign asbestos pleural plaque as opposed to mesothelioma alone. The larger specimens also provided an added degree of certainty in diagnosis. In three cases the specimens obtained with the rigid thoracoscope changed the diagnosis from reactive pleurisy to probable mesothelioma in two and to "suspicion of malignancy" in one. On the other hand, the fibreoptic instrument provided better biopsy material in three cases: caseating granulomas in one, adenocarcinoma in another, and malignancy, probably mesothelioma (rather than suspicion of malignancy), in the third.

The rigid thoracoscope was easier to manipulate as the flexibility of the fibreoptic bronchoscope made orientation and the taking of biopsy specimens difficult. This problem was overcome by passing the bronchoscope through the trocar of the Storz instrument but after 10 examinations this had resulted in minor damage to the rubber seal at the tip of the instrument from friction against the metal rim of the trocar. Although easily repaired, this could have allowed sterilising fluid access to the fibreoptic bundles or the mechanical components of the instrument. Senno $e t a l^{11}$ suggested passing the fibreoptic instrument through an Argyle intercostal chest drain to provide support, but we found this method to be clumsy and unsatisfactory. The problem was best overcome by ensheathing the flexible bronchoscope 
with a close fitting siliconised polyvinyl tube (internal diameter $6.5 \mathrm{~mm}$ ), which was then passed through a standard Maligar trocar.

In general, patients found that the procedure caused only minor discomfort, most considering the previously performed Abrams pleural biopsy to be more painful. Transient pain was experienced as biopsy was performed on unanaethetised pleura, although in most patients with chronic pleurisy (malignant or benign) pleural sensation appeared to be depressed, with little or no discomfort reported when visceral or parietal pleura was touched. In three patients moderate pain occurred when the parietal pleura was touched and sharp transient pain when the biopsy specimen was taken; the pleurisy was relatively acute in these three patients (all with benign conditions) and presumably pleural sensation was retained or increased.

Lung deflation was surprising well toleratedpresumably because the effusion had already caused partial lung collapse, so that further deflation was barely felt, and possibly because of improved matching of ventilation and perfusion with the dependent ventilated lung better perfused. In some patients adhesions prevented total lung collapse. In one case of tuberculous pleurisy associated with a small effusion the lung collapsed rapidly and completely when the pleural cavity was opened, causing dyspnoea and chest discomfort; this settled after a few minutes when the patient breathed oxygen. Supplementary oxygen was also given to five patients with arterial hypoxaemia. The ear oximeter was available for use in only six patients (all air breathing), and these patients showed only minor falls in oxygen saturation, the lowest value recorded being $89 \%$. The maximum fall in oxygen saturation of $4 \%$ occurred in two patients on induction of the pneumothorax. Lung expansion at the end of the procedure was usually rapid and was confirmed radiologically before removal of the tube in the first five patients. As in previous studies, ${ }^{56815}$ there were no instances of persistent air leak despite biopsy of the visceral pleura in five cases, in one of which the specimen included lung tissue. Tetracycline pleurodesis was attempted in 17 patients and it successfully controlled recurrent effusions in 14 , the three failures being in cases where full lung reexpansion could not be achieved.

Complications consisted of two cases of tumour growth in the wound and one minor wound infection. The tumour growth in the wound was asymptomatic in one patient and responded to local radiotherapy in the other. No cardiac arrhythmias occurred. There was one late pleural space infection, but this occurred six weeks after thoracoscopy in association with a malignant bronchopleural fistula and is unlikely to have been related to the thoracoscopy.

\section{Discussion}

The first aim of this study was to assess the practicability of thoracoscopy performed by respiratory physicians. For the procedure to be acceptable it $\vec{\nabla}$ should be relatively simple, effective, and safe. In our $\cong$ hands thoracoscopy under local anaesthesia was is acceptable to the patients, causing little more dis- $\vec{\circ}$ comfort than insertion of a chest drain or blind pleural $\rightarrow$ biopsy. Local anaesthetic needs to be generously $\vec{\omega}$ infiltrated into a fairly large area at the site of insertion so that movement of the instrument is not uncom- $\vec{x}$ fortable. An alternative would be the use of an $\vec{\omega}$ intercostal nerve block, perhaps in addition to infiltration at the insertion site. Taking biopsy specimens from pleura distant to the insertion site caused only 0 transient pain. In the occasional patient who complains of severe discomfort local anaesthetic could be $\vec{\nabla}$ infiltrated via the injecting needle provided for the Storz thoracoscope, or the cytology aspiration needle in the case of the bronchoscope; but we have not done $\vec{\oplus}$ this. Previous studies on large numbers of patients $\infty$ confirm the acceptability of performing thoracoscopy with local anaesthesia. ${ }^{47911}$

Thoracoscopy is of proved value in cases of pleural effusion of unknown cause and, unlike blind pleural biopsy, may provide reassurance in case of benign $\stackrel{\mathbb{Q}}{\stackrel{2}{2}}$ chronic pleurisy. We found that the thoracoscopic $\overrightarrow{\vec{F}}$ appearances were not always confirmed histologically. $\frac{\circ}{3}$ Although Enk and Viskum ${ }^{6}$ reported that the thoracoscopic appearances were reliable, other studies have pointed to the danger of reliance on inspection alone. ${ }^{57}$ The diagnostic yield and false negative rate in 0 the present series compares well with those of previous reports, ${ }^{24-689}$ especially as our criteria for thoraco- 0 scopy were more stringent than in previous surgical series. The high incidence of mesothelioma is a reflection of the difficulty in establishing this diagnosis from the small fragments usually obtained with the 의 Abrams needle ${ }^{16}$ and of the high frequency of asbestos $\supset$ exposure in our patients. In this disease thoracoscopy을 is frequently diagnostic, has a lower morbidity than open thoractotomy, ${ }^{17}$ may give prognostic infor- 0 mation, ${ }^{1819}$ and may be combined with a palliative $\tilde{O}$ pleurodesis; it may also allow patients to obtain $N_{\omega}$ compensation. Without histology, treatable conditions may be overlooked, as in our patient witho tuberculosis wrongly suspected of having a mesoth- $\overline{\mathbb{D}}$ elioma. Tumour seeding in the wound may be pre- + vented by routine radiotherapy, ${ }^{8}$ but the incidence of 0 this complication has probably been overstated in the past. ${ }^{19}$ It developed in two of our patients, responding well to radiotherapy in the one who had symptoms $\Omega$ from it.

Other complications were minor in the present series, the late pleural infection almost certainly beingo 
unrelated to the procedure itself. Enk and Viskum ${ }^{6}$ reported few complications in a series of 556 thoracoscopies performed with local anaesthesia: 39 cases of surgical emphysema, two episodes of vasovagal collapse, and one case of non-fatal air embolism. A recent review ${ }^{15}$ of the reported complications estimated, on the basis of over 8000 thoracoscopies, that empyema and haemorrhage requiring treatment should be expected in around $1 \%$ of cases.

The routine use of tubal drainage at the end of the procedure is unnecessary, as a persistent air leak is uncommon even when biopsy is performed on visceral pleura; moreover, it increases the risk of infection, 515 as does incomplete lung expansion. Subcutaneous emphysema occurs more frequently, in up to $7 \%$ of cases, without drainage but only one death has been reported, a result of massive mediastinal and subcutaneous emphysema. ${ }^{15}$ Respiratory insufficiency during thoracoscopy has not been mentioned as a complication in previous series and the level of pulmonary function required for safe thoracoscopy has not been assessed. ${ }^{15}$ We provided supplementary oxygen via nasal cannulas to five patients with arterial hypoxaemia. We could monitor arterial oxygen saturation in only six patients but our findings are in agreement with those of Oldenburg and Newhouse, ${ }^{7}$ who recorded a mean fall in oxygen saturation from $93 \%$ (SD 5\%) to $91 \%(4 \%)$ in 12 patients, although they reported further transient desaturation (lowest oxygen saturation $83 \%$ ) after coughing or breath holding. In the absence of appreciable carbon dioxide retention it might be wise to use supplementary oxygen routinely. As in previous studies, ${ }^{715}$ no cardiac arrhythmias were observed in our patients.

We found that the inherent flexibility of the fibreoptic bronchoscope made examination of the thoracic cavity more difficult than with the rigid instrument, whereas the skill of rigid thoracoscopy was easily learnt. The bronchoscope was made more acceptable by providing external support, the most suitable being close fitting PVC tubing as a metal trocar damaged the bronchoscope. The flexible instrument has a range advantage over the Storz instrument, making access to the apex and paravertebral area easier. Some operators use the longer laparoscope for this reason. ${ }^{20}$ There was little to choose between our two instruments in terms of optical image, although we preferred the higher optical resolution of the fibreoptic image. Some difficulty was experienced in obtaining biopsy specimens of pleural plaques with the smaller bronchoscopy forceps, and it was generally necessary to manoeuvre the bronchoscope so that the forceps were at a $90^{\circ}$ angle to the pleura to prevent the forceps slipping. It therefore took longer to obtain a specimen, but in no case were we unable to do so. Possibly the smaller forceps used with the Olympus $2 \mathrm{BF}$ in- strument resulted in the failure of Oldenburg and Newhouse ${ }^{7}$ to obtain pleural biopsy specimens in two cases.

The initial impression of the diagnostic adequacy of the biopsy specimens obtained with the two instruments was that there were no essential differences. When a second pathologist, however, was asked to report on the specimens obtained with the rigid thoracoscope and the fibreoptic bronchoscope mixed in random order, the specimens from the rigid instrument were generally preferred, although occasionally the bronchoscopic specimens were more informative. The difficulty associated with the smaller bronchoscopic specimens is analogous to the early experience with rigid and fibreoptic bronchoscopy. ${ }^{21} 22$

In conclusion, we have confirmed the acceptability of "medical" thoracoscopy. It is a technique that is easily learnt, safe, and practical with local anaesthesia, and can readily be performed by interested respiratory physicians. The rigid thoracoscope is a more satisfactory instrument but with minor adaptations the flexible bronchoscope makes an acceptable alternative if a rigid thoracoscope is unavailable. Thoracoscopy should be considered early in the course of investigation of a patient with a pleural effusion, possibly in place of Abrams biopsy if simple aspiration is not diagnostic.

We express our appreciation to $\mathrm{Mr}$ Buckley for technical assistance and to Pentax for providing the bronchoscope and the PVC tubing.

\section{References}

1 Jacobaeus HC. Possibility of the use of cystoscope for investigation of serous cavities. Munch Med Wochenschr 1910;57:2090.

2 Bloomberg AE. Thoracoscopy in perspective. Surg Gynecol Obstet 1978;147:433-43.

3 Brandt HJ, Mai J. Differential diagnosis of pleural effusion using thoracoscopy. Pneumonologie 1971; 145:192-203.

4 Decamp PT, Moseley PW, Scott ML, Hatch HB. Diagnostic thoracoscopy. Ann Thorac Surg 1973;16:79-84.

5 Canto A, Blasco E, Casillas M, et al. Thoracoscopy in the diagnosis of pleural effusion. Thorax 1977;32:550-4.

6 Enk B, Viskum K. Diagnostic thoracoscopy. Eur J Respir Dis 1981;62:344-51.

7 Oldenburgh FA, Newhouse MT. Thoracoscoy. A safe, accurate diagnostic procedure using the rigid thoracoscope and local anaesthesia. Chest 1979;75:45-50.

8 Boutin C, Viallat JR, Cargnino P, Farisse P. Thoracoscopy in malignant pleural effusions. Am Rev Respir Dis 1981;124:588-92.

9 Swierenga J, Wagenaar JPM, Bergstein PGM. The value of thoracoscopy in the diagnosis and treatment of diseases affecting the pleura and lung. Pneumonologie 
1974;151:11-8

10 Gwin E, Pierce G, Boggan M, et al. Pleuroscopy and pleural biopsy with the flexible firbreoptic bronchoscope. Chest 1975;67:527-31.

11 Senno A, Moallem S, Quijano ER, et al. Fibreoptic thoracoscopy. NY State J Med 1975;75:51-6.

12 Lewis RJ, Kunderman PJ, Sisler GE, Mackenzie JW. Direct diagnostic thoracoscopy. Ann Thoracic Surg 1976;21:536-9.

13 Baumgartner WA, Mark JBD. The use of thoracoscopy in the diagnosis of pleural disease. Arch Surg 1980; 115:420-1.

14 Ayliffe GAJ, Coates D, Hoffman PN. Chemical disinfection in hospitals. Colindale: Public Health Laboratory Service, 1984:20-2.

15 Viskum K, Enk B. Complications of thoracoscopy. Poumon Coeur 1981;37:25-8.

16 Whitaker D, Shilkin KB. Diagnosis of pleural malignant mesothelioma in life - a practical approach. J Pathol
1984;143:147-75.

17 Edge JR, Choudbury SL. Malignant mesothelioma of the $\stackrel{\vec{F}}{\rightarrow}$ pleuta in Barrow-in-Furness. Thorax 1978;33:26-30.

18 Boutin C, Farrisse P, Viallat JR, Carginino P, Choux R. 흐 La thoracoscopie dans le mesotheliome pleural. $\operatorname{Rev} \operatorname{Fr} \overline{\mathrm{S}}$ Mal Respir 1979;7:680-6.

19 Law MR, Hudson ME, Heard BE. Malignant mesothelioma of the pleura: relation between histological type $\sqrt{s}$ and clinical behaviour. Thorax 1982;37:810-5.

20 Sang CTM, Braimbridge MV. Thoracoscopy simplified using the laparoscope. Thorac Cardiovasc Surg $\vec{\omega}$ 1981;29:129-30.

21 Webb J, Clarke SW. Comparison between the positive $\vec{x}$ biopsy rates in lung cancer achieved with the rigid bronchoscope and the fibreoptic bronchoscope. Thorax 1978;33:531-2.

22 Knight RK, Clarke SW. An analysis of the first $300 \mathrm{~N}$ fibreoptic bronchoscopies at the Brompton hospital. $\mathrm{Br} O$ J Dis Chest 1979;73:113-20.

\section{Book notices}

Abnormalities of Respiration during sleep. E C Fletcher. ( $\mathrm{Pp}$ 252; \$39.50.) Orlando; Florida: Grune and Stratton, 1986. ISBN 0-8089-1812-5.

There has been a rush of books on sleep (and breathing) disorders over the last two years and these are probably the best:

1 Saunders NA, Sullivan CE, eds. Sleep and breathing. New York: Marcel Dekker, 1984.

2 Kryger MH, ed. Clinics in Chest Medicine: Symposium on sleep disorders. Philadelphia: WB Saunders, 1985.

3 Thawley SE, ed. Medical Clinics of North America: Symposium on sleep apnea disorders. Philadelphia: WB Saunders, 1985.

4 Parkes JD. Sleep and its disorders. London: WB Saunders, 1985.

The first three of these accounts tend to be mainly from North American sources and thus contain extensive reviews of the published work with little interpretation or "clinical feel." The collection of Saunders and Sullivan remains the definitive academic text at present but this new book from Eugene Fletcher is a much better and shorter account for clinicians coping with sleep and breathing disorders. Despite the clinical flavour no really important academic aspects are ignored. The best chapters, by Fletcher himself, are very readable and up to date, and analyse and comment rather than just recount, and they include, for example, case histories to give a good clinical feel to the subject. It is a pity that Fletcher did not also write the "Clinical manifestations of sleep apnoea" chapter as well. The "Mechanisms of sleep apnoea" chapter by Kuna and Remmers is a very balanced account, as is the description of surgical treatments by Cohn. I can thoroughly recommend this book as a sensible and careful account of a subject that has unfortunately gained a reputation for extravagance of claims in both diagnosis and $\vec{\oplus}$ treatment. This and Parkes's book recounting non- $\infty$ respiratory sleep disorders together cover best this rapidly growing area for European readers and will be appreciated by both newcomers and old hands in the subject. - JRS

Respiratory Disease. A E Tattersfield and M W McNicol. (Pp 288; £14.95.) London: Springer Verlag, 1987. ISBN 3-540- $\overline{0}$ 16209-7.

This book is one of a series entitled "Treatment in Clinical Medicine." I found it to be both informative and wide ranging in its content, with adequate references for the $\overline{0}$ inquiring reader. The title is perhaps misleading as almost two thirds of the book deals with basic mechanisms, pathophysiology, and treatment of the whole range of respiratory 3 disorders in a concise and up to date fashion, and in a conventional way much as is found in other texts. The final $O$ third of the book discusses the pharmacological, therapeutic,

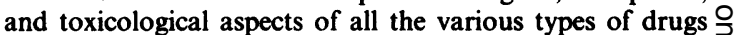
which a respiratory physician would use with the exception of $D$ cytotoxic agents, which are not discussed in detail. I believe that the book could usefully have included more in this area, particularly on the general side effects and those associated $\sigma$ with specific drugs. Otherwise, the detail and scope of $N$ discussion in this latter section is far greater than that found $\mathrm{N}$ in existing texts, and this would be useful for the respiratory $\omega$ specialist. The arrangement of the book is a little awkwardfor example, to read about the treatment of asthma the readere would be required to refer to chapter 4 for a generalised $\overparen{D}$ outline of management and subsequently to chapters $14-19 \stackrel{\oplus}{+}$ for a more detailed consideration of the various drugs $\square$ involved. This inevitably leads to some repetition. Overall 0 this is an interesting text that usefully fills a gap in the existing $\overrightarrow{\mathbb{D}}$ range. In their preface the authors suggest that it is most $\frac{}{\mathbb{D}}$ suitable for those beginning to specialise in respiratory 2 medicine and for such individuals I strongly recommend it. It $\bar{\gamma}$ is not, however, a book aimed at the undergraduate unless he $?$ has a particular interest in therapeutics.-CRS 